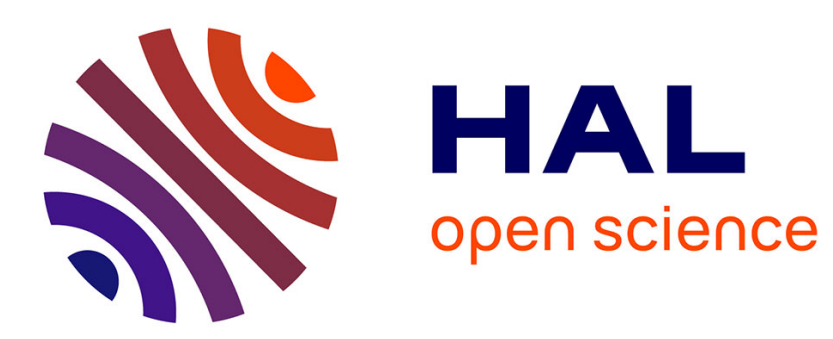

\title{
Une perspective anthropologique sur l'infanticide: la notion de personne en Nouvelle-Guinée
}

Pascale Bonnemère

\section{To cite this version:}

Pascale Bonnemère. Une perspective anthropologique sur l'infanticide: la notion de personne en Nouvelle-Guinée. Enfances \& Psy , 2009, 44, pp.29-41. 10.3917/ep.044.0029 . hal-00439060

\section{HAL Id: hal-00439060 https://hal.science/hal-00439060}

Submitted on 13 Feb 2020

HAL is a multi-disciplinary open access archive for the deposit and dissemination of scientific research documents, whether they are published or not. The documents may come from teaching and research institutions in France or abroad, or from public or private research centers.
L'archive ouverte pluridisciplinaire HAL, est destinée au dépôt et à la diffusion de documents scientifiques de niveau recherche, publiés ou non, émanant des établissements d'enseignement et de recherche français ou étrangers, des laboratoires publics ou privés. 


\title{
Une perspective anthropologique sur l'infanticide : la notion de personne en Nouvelle-Guinée
}

\author{
Pascale Bonnemère ${ }^{1}$
}

De tous les termes qui désignent l'acte de donner la mort, « infanticide » est sans doute le plus difficile à entendre. Serait-ce parce qu'il évoque le meurtre d'un être sans défense ? Ou parce qu'il est le plus souvent perpétré par celle-la même qui a donné la vie ? Les questions qu'un tel acte soulève sont en tout état de cause de divers ordres et les différentes sciences humaines qui sont à même de l'étudier (la psychologie, l'histoire et l'anthropologie essentiellement) le font chacune à sa manière. En tant qu'anthropologue, il ne me revient pas, par exemple, de considérer l'infanticide comme un acte exprimant une pathologie mentale ou reflétant une défaillance dans le lien mère-enfant. La réflexion anthropologique sur la mise à mort d'un nouveau-né doit avant toute chose replacer ce geste extrême dans le contexte social au sein duquel il a lieu. Autrement dit, l'infanticide ne peut être appréhendé en dehors du système d'organisation de la parenté, des codes moraux en vigueur et des représentations de la personne dans la société particulière où il est observé.

À écouter les Ankave de Papouasie-Nouvelle-Guinée chez lesquels je mène des enquêtes ethnographiques depuis un peu plus de vingt ans, il n'y aurait existé qu'une femme infanticide, qui, dit-on, tuait à la naissance tous les enfants qu'elle mettait au monde, sans que personne d'autre qu'elle-même ne les voie jamais. Jusqu'à son décès, en 2000, Madibi a toujours évité ma présence, sans doute parce que, tout en étant membre d'une société qui ne condamne pas socialement un tel acte - le tribunal villageois qui siège lorsque survient un conflit grave ne s'est pas réuni pour discuter des soupçons qui pesaient sur elle -, elle avait néanmoins conscience du caractère non anodin de son geste. D'autres femmes ont qualifié parfois, mais du bout des lèvres seulement, son comportement vis-à-vis des enfants qu'elle portait comme étrange et anormal, mais le sujet ne les intéressait guère, comme s'il ne leur revenait pas le droit de condamner un acte dont j'ai le sentiment qu'elles le percevaient avant tout comme une décision éminemment individuelle. Même son mari ne semblait pas avoir eu son mot à dire. Elle était par ailleurs considérée par tous comme une bonne épouse, autrement dit quelqu'un qui soignait ses jardins, tenait correctement sa maison et préparait les repas de son mari.

Ne disposant pas de matériaux ethnographiques de première main à présenter sur un tel phénomène, qui de surcroît revêt un caractère tout à fait idiosyncrasique chez les Ankave, il m'a fallu chercher dans la littérature anthropologique des éléments permettant de mieux comprendre les contextes sociaux et symboliques dans lesquels l'infanticide n'est pas considéré comme un acte moralement répréhensible ${ }^{2}$. De toute évidence, la définition de ce qui relève d'un crime et de ce qui n'en relève pas dépend étroitement du statut accordé à la personne qui en est victime. Cette remarque ne vaut pas seulement pour les crimes de nouveau-nés, même s'ils revêtent dans ce cas une allure toute particulière, le statut des bébés variant éminemment d'une société à une autre.

1 Pascale Bonnemère est directrice de recherche au CNRS, membre du Centre de Recherche et de Documentation sur l'Océanie (CREDO, Marseille).

${ }^{2}$ Comme le lecteur s'en rendra vite compte, le présent article se fonde essentiellement sur une ethnographie consacrée à la Nouvelle-Guinée, avec néanmoins de rares incursions ailleurs, y compris dans notre propre passé. 
En Occident, ce n'est pas sur le statut du nouveau-né mais sur celui de l'embryon que le consensus fait défaut, comme le révèlent les débats, souvent vifs, à propos de la légalisation de l'avortement. Certains le considèrent comme une personne dès la conception et font par conséquent de l'avortement un crime, d'autres ne voient pas de problème moral à se débarrasser d'un fœutus, du moins en deçà d'un certain nombre de semaines d'aménorrhée. Parmi les pays qui ont autorisé l'avortement, la disparité des législations à propos de ce délai illustre bien le fait que considérer comme acceptable l'interruption d'une grossesse à tel ou tel moment ne vas pas de soi. À l'échelle de la planète, l'infanticide soulève le même type de questionnements que l'avortement dans les pays occidentaux. Commentant les matériaux ethnographiques recueillis par T. Gregor chez les Indiens Mehinaku de l'Amazonie brésilienne, qui montrent que, là-bas, les femmes voient et traitent certains de leurs nouveau-nés comme des pré-humains, l'anthropologue N. Scheper-Hugues considère «le néonaticide comme une forme d"avortement post-partum» (1992, p. 433). La principale question qui sous-tend la compréhension d'un acte tel que l'infanticide dans une société donnée est par conséquent : à partir de quel moment un nouvel être humain est-il localement considéré comme une personne ? La réponse varie selon que l'on habite un pays européen ou une vallée reculée de Papouasie-Nouvelle-Guinée. Dans une société où un nouveau-né n'est pas considéré comme une personne à part entière, décider de le tuer pose probablement moins de difficultés morales que dans celle où un embryon de quelques semaines est appréhendé comme une personne en devenir, et cela même si les sentiments ressentis par les femmes infanticides sont sans doute aussi pleins d'ambivalence et de doute que partout ailleurs (Scheper-Hughes, 1992, p. 432).

II semble également difficile de comparer un phénomène comme l'infanticide dans une région du monde où les femmes ont accès à la contraception, peuvent choisir d'allaiter ou non leurs enfants et sont à même de leur apporter tous les soins dont ils ont besoin et dans une autre, comme la Nouvelle-Guinée, où la très grande majorité des habitants vivent en milieu rural, n'ont qu'un accès très limité aux services de santé et ne disposent ni de moyens de contraception ni de laits de substitution au lait maternel. Certes, il s'agit du même acte mais le parallèle s'arrête là tant la signification du geste dépend, outre du système de représentations mentales de ce qu'est une personne, du contexte social et médical dans lequel il prend place.

\section{Quand la naissance ne suffit pas à faire d'un nourrisson une personne}

Pour certains anthropologues, l'infanticide doit être appréhendé comme un moyen de réguler les naissances ou d'opérer une sélection naturelle. Il est alors considéré au sein d'un continuum de comportements affectant la fertilité et concourant au contrôle de la taille de la famille : entre l'évitement de la conception et le meurtre d'un nourrisson ou d'un jeune enfant, il n'existerait donc qu'une différence de degré (Scrimshaw, 1984, p. 440). Dans des situations où les ressources alimentaires ne sont pas suffisantes ou risquent de ne pas l'être un jour, les nouvelles bouches à nourrir seraient éliminées (Scrimshaw, 1984, p. 453). Selon ce point de vue, l'infanticide des filles présente un avantage puisqu'un nombre limité d'hommes pourrait suffire à assurer la reproduction, et cela même en tenant compte du fait que le nombre d'enfants qu'une femme peut engendrer pendant la durée de sa vie fertile n'est pas infini (Scrimshaw, 1984, p. 454). Ce raisonnement a servi dans l'étude de l'infanticide chez les Eipo Mek, une société de Papouasie occidentale, la province indonésienne située dans la partie orientale de l'île de Nouvelle-Guinée. Selon l'ethnographe de ce groupe, Wulf Schiefenhövel, l'infanticide constituait dans les années 1970 un moyen courant et non problématique de limiter la croissance démographique dans un environnement difficile (Butt, 1998, p. 157). Dans ce type de sociétés, la pratique de l'infanticide serait rendue culturellement et individuellement acceptable grâce à une définition de la vie selon laquelle un enfant ne devient pas membre de sa société aussitôt qu'il est né mais après un certain laps de temps (Scrimshaw, 1984, p. 460-461). 
Tout en contestant les explications des meurtres de nouveau-nés que prône cette anthropologie qui voit dans tout produit de l'action humaine l'expression d'un comportement adaptatif, on ne peut que constater que, dans bien des populations, la naissance ne suffit pas à faire d'un nourrisson une personne. II nous faut donc revenir à ce champ d'investigation-là. C'est d'ailleurs ainsi que l'historienne A. Tillier termine son ouvrage sur les femmes infanticides en Bretagne entre 1825 et 1865 : " L'origine rurale des criminelles, pour qui la mise à mort des animaux est un acte banal, la familiarité des hommes du XIXe siècle avec la mort, l'importance de la mortalité infantile atténuent sans doute à leurs yeux la portée de leur acte. D'autant que pour nombre d'entre elles le nouveau-né n'est pas encore une personne » (2001, p. 400). II s'agit de la toute dernière phrase du livre, qui reflète parfaitement les situations que les anthropologues rencontrent.

Pour les Ankave de Papouasie-Nouvelle-Guinée, par exemple, les nouveau-nés ne manifestent pas de capacités relationnelles, ils ne sourient pas volontairement, leur regard est absent et il faut attendre plusieurs mois pour qu'ils acquièrent une certaine aptitude à entrer en relation avec autrui. Cette aptitude nécessite l'acquisition du denge', une entité spirituelle qui permet à l'enfant d'être attentif, de porter son regard sur un objet précis, de sourire, de reconnaître sa mère. Or le denge' n'est pas développé chez les nourrissons et ne se manifeste qu'au bout de plusieurs mois de proximité avec les êtres humains, et avec sa mère en premier lieu. Cet esprit denge' est volatil, comme l'atteste la pratique de lier lâchement les poignets des bébés pour ne pas qu'il s'échappe lorsque, en se réveillant, ils écartent brusquement les bras. Dans les propos des Ankave sur les nouveau-nés, c'est le caractère vulnérable de ceux-ci qui domine : une femme qui vient d'accoucher, en position accroupie, ne touche en effet pas son nourrisson ; celui-ci voit le jour sur une natte en écorce battue qu'elle a placée sous elle. Et ce n'est qu'après avoir effectué une magie appropriée qu'elle pourra le prendre dans ses bras sans risque pour sa croissance. Pour la même raison, son père ne le touchera qu'une fois qu'il aura été lavé pour la première fois, ce qui ne se passe pas avant plusieurs jours, quand ce n'est plusieurs semaines. Les hommes ajoutent que, du fait qu'il est sorti du corps d'une femme, un bébé est une source de pollution et qu'il ne serait pas sans danger pour eux d'entrer trop tôt en contact avec lui. En raison de la vulnérabilité de leur denge', qui est encore en formation, et de celle de leur corps au contact des adultes, les nourrissons sont des êtres considérés comme très fragiles, ce que confirmerait le taux de mortalité infantile extrêmement élevé que j'ai calculé à partir des récits de vie des femmes de la vallée. Aujourd'hui encore, seul un bébé sur trois survit en effet à sa première année.

La fin de la première année est à peu près le moment où un bébé est doté d'un nom personnel qu'il conservera toute sa vie. Mais plutôt que de voir dans ce processus tardif le délai nécessaire pour s'assurer que le bébé survivra, il paraît plus conforme à la réalité de l'attribuer au fait que les nourrissons sont considérés comme des êtres encore relativement indifférenciés les uns des autres, qu'il va falloir progressivement intégrer au clan paternel et à la société dans lesquels ils sont nés. Considérés comme les produits du clan maternel en ce que la mère est celle dont le sang fait grandir le fœetus, ils ne deviendront en effet membres du clan de leur père - le système de filiation est ici patrilinéaire - qu'après que celui-ci a offert de la nourriture et versé une compensation matrimoniale à la famille de son épouse. Cette inscription dans le tissu social de la parenté ne s'opère pas à la naissance mais seulement une fois que le bébé aura démontré son aptitude à réagir à la présence d'autrui et à interagir avec lui. Le critère permettant d'accorder le statut de personne à un être humain est donc avant tout sa capacité à entrer en communication avec les autres, à répondre à leurs sollicitations, à chercher à attirer leur attention. Mais l'enfant de sexe masculin devra encore passer par toute une série d'étapes, la plupart ritualisées, pour devenir une personne adulte ${ }^{3}$.

${ }^{3}$ Les jeunes garçons ankave doivent subir un certain nombre d'épreuves initiatiques pour parvenir à maturité. Elles s'organisent en trois phases : deux collectives, lorsqu'ils sont âgés 


\section{Les nouveau-nés korowai : des êtres ambigus}

Dans une autre société de l'île de Nouvelle-Guinée, les Korowai, qui vivent dans sa partie occidentale, province indonésienne, les nouveau-nés représentent une altérité radicale (2009, p. 152). Lorsqu'une femme korowai ressent les premières douleurs de l'enfantement, son mari creuse un trou de la taille d'un enfant dans le sol de l'endroit choisi pour l'accouchement, toujours situé aux marges du village (2009, p. 150). La femme donne naissance à son bébé en s'accroupissant au-dessus de ce trou. Dans le passé, une proportion relativement importante d'enfants n'en étaient pas extraits pour être emmenés au village et intégrés à la communauté mais étaient étouffés - on enfonçait un paquet de feuilles dans leur bouche - et enterrés sur place $(2009, \text { p. 151 })^{4}$. À partir d'histoires de vie recueillies auprès de soixante-dix femmes, l'ethnographe estime à $40 \%$ le pourcentage de nourrissons ainsi éliminés. Le sexe de l'enfant n'entrait apparemment pas en ligne de compte dans la décision de le garder en vie ou non ${ }^{5}$.

Avant d'être en contact avec des missionnaires et des agents de l'État indonésien, les Korowai ne voyaient pas dans l'infanticide une pratique susceptible de poser un quelconque problème moral, essentiellement en raison du fait que, pour eux, les nouveau-nés ne sont pas considérés comme des êtres humains. Ils appartiennent à la catégorie des démons, les laleo, dans laquelle sont rangés des êtres malveillants qui se manifestent sous la forme de cadavres ambulants aux allures de zombies. Plus généralement, le terme est utilisé pour désigner des entités présentant des anomalies ou des caractéristiques étranges. Les nouveau-nés sont donc appréhendés comme des êtres profondément différents de tout un chacun, des sortes d'aliens associés à la condition des défunts plutôt qu'à celle des êtres humains vivants $(2009$, p. 152). On dit des bébés qu'ils acquièrent un corps d'humain au bout d'un mois ou deux, lorsque leur peau s'assombrit et qu'ils commencent à réagir à la présence d'autrui (2009, p. 151).

Les nouveau-nés korowai dont le destin était de mourir n'étaient pas même touchés : ils étaient étouffés là où ils étaient nés, dans ce trou qui deviendrait leur tombe, encore reliés au placenta. On justifiait cette absence de contact en partie par la souillure que provoquent les substances libérées à la naissance et en partie par la qualité prétendue démoniaque des nourrissons. Mais elle était également le signe qu'aucune relation sociale et morale n'allait être établie entre ceux-ci et leurs mères, et avec les êtres humains en général (2009, p. 150). Pour ceux des enfants qui seraient intégrés à la communauté, le premier acte marquant la volonté d'en prendre soin était la coupure du cordon ombilical. Le second geste témoignant de l'entrée du nouveau-né dans une amorce de relation sociale était de le placer dans le creux d'une feuille de palmier-sagoutier et de l'emporter chez soi ${ }^{6}$.

d'une dizaine d'années et la dernière, individuelle, lorsque leur premier enfant vient de naître. On trouvera des détails sur ces rituels dans Bonnemère 1998 et 2008, entre autres références.

${ }^{4}$ Sur l'infanticide par étouffement, voici ce qu'écrit L. Boltanski dans La condition fœtale : « La destruction a lieu immédiatement après la sortie du nouveau-né de la matrice, souvent par étouffement. Aucun rituel de passage de la vie à la mort n'accompagne cet acte. On fait simplement disparaître le corps. Accomplie à l'écart de la vie publique, l'élimination n'a pas à être justifiée. On fait comme si l'enfant n'était pas né et donc comme si la destruction n'avait pas eu lieu » $(2004,64)$.

5 Alors que, dans certains pays (la Chine et I'Inde par exemple), l'infanticide - ou l'avortement aujourd'hui qu'il existe l'échographie - ne concerne que les filles, aboutissant à un « sex ratio » qui deviendra vite problématique en matière matrimoniale (Le Monde du 20 mai 2009) ; en Nouvelle-Guinée le fait n'est pas significatif, je ne m'y attarde donc pas ici.

${ }^{6}$ On rappellera ici les pratiques de la Rome ancienne : " II ne suffit pas de naître, dans une famille romaine, pour y être élevé : le bébé doit être soulevé (tollere) de terre où l'a déposé la 
Pour Rupert Stasch, l'ethnographe des Korowai, le choix de garder ou de se débarrasser d'un nourrisson n'était pas fait à l'avance mais s'opérait lors de la naissance. Ce moment est empreint de sentiments profondément négatifs associés à la maladie, la mort et l'inhumanité (2009, p. 150). La coupure du cordon ombilical permet la séparation du nouveau-né d'avec le placenta, source de maladie et de mort, et son éloignement du passage direct de la matrice maternelle à la tombe. Un tel geste est interprété comme une façon de rendre un peu humain l'être qui vient de naître.

Quelles sont les raisons données par les Korowai pour justifier les infanticides qu'ils pratiquaient dans un passé encore récent? La charge lourde que représente l'éducation des enfants, dans un contexte où la nourriture vient parfois à manquer et où la place des petites communautés est perçue comme incertaine dans un monde en transformation, figure parmi les premiers motifs justifiant de ne pas laisser vivre tous les enfants qui viennent au monde (2009, p. 150). Parfois, ce sont des circonstances sociales particulières qui sont invoquées: le père n'a pas encore versé la compensation matrimoniale qu'il doit à la famille de son épouse ou le bébé est le fruit d'une relation adultère ${ }^{7}$. Dans le cas où le père est décédé pendant la grossesse, le risque que le nouveau-né soit une manifestation de la présence du défunt est tellement fort que sa mère préfère l'éliminer.

Plus généralement, les propos des Korowai laissent transparaître une profonde ambivalence entourant tout ce qui relève de la reproduction : les substances libérées lors d'un accouchement leur inspirent une répulsion extrême et ils considèrent le contact avec les déchets corporels des bébés (urine, feces) comme particulièrement désagréable. À cette répulsion devant la physiologie de la reproduction s'ajoute l'idée que porter et élever des enfants accélère le processus de vieillissement. Pour les Korowai, avoir des enfants comporte une dimension éminemment sacrificielle (abnégation, vieillissement accéléré, etc.) qu'ils ont parfois du mal à accepter et ils évoquent fréquemment ce caractère asymétrique des relations parents-enfants (2009, pp. 155-156).

Mais par-dessus tout, ce qui rend l'infanticide possible et moralement acceptable dans cette société, c'est le statut qui est accordé au nouveau-né. Comme on l'a vu, celui-ci n'est pas encore humain et il présente un caractère d'étrangeté l'apparentant à une figure démoniaque. En tout état de cause, la décision de sauver un enfant de l'infanticide n'est jamais prise en raison d'attributs qu'il a en tant que nouveau-né, mais toujours en fonction de ce qu'il deviendra (2009, p. 144). Comme le disent les Korowai, "les enfants n'ont pas de proches", ce qui signifie que, dans cette société, ils ne sont pas apparentés à leurs ascendants en vertu d'un lien généalogique mais le deviennent au travers des interactions qu'ils ont avec eux. En quelque sorte, ils ne sont pas intégrés à un réseau social qui leur préexiste, mais celui-ci doit se construire au travers de relations sociales réelles. L'allaitement est pour une mère le premier de ces actes concourant à l'établissement de relations sociales avec son nouveau-né et au processus qui va faire progressivement de lui une personne (2009, p. 167).

sage-femme, puis pris dans les bras par son père. Ce geste signifie que le père reconnaît son fils, s'engage à le nourrir et en même temps établit ses droits sur lui. Si l'enfant est une fille, le père ordonne seulement de l'alimenter. Dans le cas contraire, le bébé est exposé sur la voie publique, étouffé ou simplement privé d'aliments " (Valette-Cagnac, 2003, p. 50).

${ }^{7}$ Comme le montre Annick Tillier à propos de la Bretagne du XIX ${ }^{\mathrm{e}}$ siècle, les motifs d'ordre social étaient à l'origine de tous les infanticides commis par les femmes (des célibataires en très grande majorité, mais aussi quelques femmes mariées adultères ou veuves) : "les premières victimes des infanticides sont des enfants naturels » $(2001$, p. 9). 
En résumé, ce qui ressort clairement d'une lecture de travaux ethnographiques portant sur des sociétés où l'infanticide existe, c'est que ces êtres que l'on y tue ne sont jamais considérés comme des personnes. Cela n'implique aucunement que les femmes infanticides de Nouvelle-Guinée soient d'une insensibilité pathologique ou incapables de voir dans leur nouveau-né autre chose qu'un objet. Elles sont simplement - et comme il se doit - en accord avec le système de représentations mentales en vigueur dans la société dont elles sont membres. Si elles peuvent se débarrasser des êtres qu'elles viennent de mettre au monde sans éprouver de problème moral insurmontable, c'est qu'elles ne les perçoivent pas comme déjà dotés de qualités humaines.

\section{Causes et contextes des infanticides}

Le fait qu'en Nouvelle-Guinée les enfants qui présentent une malformation soient éliminés va dans le même sens. Car, comme l'écrit N. Scheper-Hugues, "le nourrisson présentant une anomalie physique congénitale met à mal les frontières symboliques fragiles entre l'humain et le non humain, le naturel et le surnaturel, le normal et l'abominable » (1992, p. 375). Chez les Ankave, lorsque l'aspect physique d'un bébé s'éloigne de la norme, ne serait-ce que par l'absence d'un orteil, l'intervention d'un esprit malveillant pendant la grossesse est suspectée et, dans la mesure où le bébé pourrait être une incarnation de cet esprit et donc un être doté de capacités surnaturelles aux intentions potentiellement destructrices, nul ne prendrait le risque de le laisser vivre. Dans le passé, un tel nourrisson était donc éliminé ; aujourd'hui, comme la croyance qu'il existe un lien entre l'intervention d'un esprit malveillant et la survenue d'une malformation physique à la naissance est moins forte, la décision de ne pas laisser vivre un nouveau-né devient une affaire d'appréciation personnelle des parents plutôt qu'une sorte d'obligation morale vis-à-vis de la communauté. Récemment, le père ankave d'une petite fille née avec le bras gauche atrophié m'a confié qu'il avait failli la tuer mais que son épouse et lui avaient finalement décidé de la laisser en vie. Quelques années plus tard, leur inquiétude initiale sur les capacités qu'aurait leur fille à accomplir les tâches domestiques quotidiennes s'était totalement dissipée tant la fillette montrait de l'habilité à se servir efficacement de son avant-bras.

Néanmoins, les raisons qui justifient un infanticide n'appartiennent pas toutes au registre de l'aspect physique des bébés qui viennent de naître, loin s'en faut. Les raisons d'ordre social, voire celles liées à des considérations économiques, prédominent même largement. Ainsi, on se débarrasse parfois d'un bébé né trop tôt après le précédent car allaiter, porter et prendre soin de deux enfants en même temps est considéré comme une charge trop lourde pour la mère. La même raison est invoquée pour expliquer que, dans le cas d'une naissance gémellaire, un seul des jumeaux soit gardé, en général celui qui paraît le plus vaillant mais parfois aussi le premier-né (Poole, 1985, p. 191). L'idée sous-jacente est que les deux auraient de grands risques de mourir et qu'il vaut alors mieux donner toutes les chances de survie à l'un d'entre eux. De même, un nourrisson dont la mère est morte en couches est rarement confié à une autre femme qui a un bébé encore au sein et donc du lait. II était parfois enterré avec elle, comme dans l'île de Dobu, en Papouasie-Nouvelle-Guinée (Brown, 1972 [1910], p. 37). En Nouvelle-Guinée, l'espacement considéré comme idéal entre deux enfants est de trois ans, le temps que dure l'allaitement, et il existe un interdit sur les relations sexuelles entre les parents d'un jeune enfant pendant cette période parce que l'on pense que le sperme gâte le lait maternel. Certains ethnologues voient dans cette pratique un des moyens utilisés pour gérer au mieux la reproduction en l'absence totale de moyens de contraception. Selon S. Lindenbaum, par exemple, croire aux pouvoirs de pollution des femmes et imposer socialement certains comportements a pour effet de poser une barrière culturelle à la reproduction humaine (1972, p. 248). Si l'interdit sur le coït est rompu et qu'une grossesse survient trop tôt, l'infanticide est alors parfois choisi. II peut être actif - le nouveauné est étouffé ou battu à mort - ou passif - la mère ne lui fournit pas les soins nécessaires à sa survie (McDowell, 1988, p. 29). 
Principale, voire unique, cause des infanticides perpétrés dans la Bretagne du XIX ${ }^{\mathrm{e}}$ siècle, étudiée par A. Tillier (2001), l'illégitimité d'un enfant né d'une union adultère est également parfois un facteur justifiant son élimination, en Nouvelle-Guinée contemporaine. La honte qu'engendre une telle naissance pour la famille de la jeune femme est la principale raison que donnaient les femmes bretonnes pour justifier leurs actes devant les juges. Enfin, les femmes de Nouvelle-Guinée usent parfois de l'infanticide comme un moyen de se venger d'un mari violent : M. Godelier, ethnographe des Baruya, voit dans cet acte extrême un acte de résistance à la domination masculine, un refus d'apporter des héritiers à un mari qu'elles ne supportent plus (1982, p. 235).

\section{Un système de représentation de la personne différent du système occidental}

Les raisons d'un infanticide peuvent donc être très variées mais, encore une fois, ce qu'il faut retenir de tous ces exemples ethnographiques, c'est que les femmes ne considèrent pas ces êtres dont elles disposent de la vie comme des personnes. Et s'il en est ainsi, ce n'est pas en raison d'un trouble d'ordre psychique ni d'une incapacité d'éprouver de l'empathie pour lui, mais parce qu'elles appartiennent à des sociétés qui ont construit un système d'idées sur la personne radicalement différent de celui rencontré en Occident. Quel est-il ? Dès les premières enquêtes ethnographiques menées dans les hautes terres de NouvelleGuinée dans les années 1950, certains ethnologues avaient perçu que les principes moraux que l'on y rencontrait révélaient une absence de valeur universelle accordée aux êtres humains comme membres de l'espèce humaine. K. Read soulignait par exemple que les Gahuku-Gama ne définissent pas d'actions qui seraient mauvaises ou bonnes dans l'absolu et pour tout le monde, mais seulement au sein d'une collectivité particulière : par exemple, alors qu'il est répréhensible de tuer un membre de son propre groupe, l'homicide à l'extérieur est parfois recommandé. II n'existerait donc pas de règle morale applicable de la même façon à tout le monde et la valeur d'une vie individuelle dépend de critères sociaux (1955, p. 262). L'individu n'est ici jamais envisageable séparément du contexte social dans lequel il évolue (1955, p. 257).

En Occident, où les représentations de la personne sont essentiellement héritées du christianisme, les êtres humains se distinguent des autres êtres vivants en ce qu'ils sont dotés d'un esprit, et les qualités de celui-ci les placent, jusqu'à un certain point, au-dessus des institutions, dans une relation d'indépendance vis-à-vis des formes sociales au sein desquelles ils vivent (Read, 1955, p. 249). La personne jouit ainsi d'une valeur absolue, qui lui est conférée non pas par sa position dans un réseau social, mais plutôt par le fait qu'elle est à l'image de Dieu (1955, p. 250). En raison de leur nature humaine, les individus sont considérés comme équivalents entre eux sur le plan moral et dotés d'une valeur unique, objective et intrinsèque et d'une individualité qui est distincte du statut qu'ils ont, des rôles qu'ils jouent et du système relationnel dans lequel ils vivent (1955, p. 275).

En Mélanésie, les choses sont différentes : l'état de personne s'acquiert de manière progressive, essentiellement dans la relation aux autres. Or, un nourrisson est un être qui, bien que théoriquement inclus aussitôt que né dans un groupe de parenté et donc en ce sens intégré à la société, n'a pas encore pu réellement entrer en relation parce que le denge', cette entité spirituelle lui permettant de reconnaître autrui, ne s'active, lentement, qu'une fois qu'il se trouve hors du corps maternel. Les exemples ethnographiques mentionnés plus haut montrent que les habitants de ces régions ont une manière très claire de lier éveil au monde des humains, capacité de communiquer et statut de personne. On serait alors tenté de dire qu'avant qu'un nouveau-né démontre une capacité d'attention se matérialisant sur son visage par des sourires et des regards, peu de choses permettent aux adultes de lui conférer un statut très différent de celui des autres êtres animés que l'on considère localement comme dotés d'une vie sociale (les chiens, les cochons, les grenouilles et les oiseaux). Dans la civilisation romaine aussi, l'enfant était rejeté du côté de 
l'animalité : « Être informe et sauvage, il n'est ni physiquement ni moralement un homme, c'est son éducation qui le fabriquera tel " ( $\mathrm{F}$. Dupont, Le citoyen romain sous la République,509-27 av. J.-C., cité par Valette-Cagnac, 2003, p. 50).

Plus généralement, comme on va le voir, dans cette région du monde les relations sont considérées comme constitutives des personnes. Chacun est venu au monde à la suite d'un mariage qui a vu deux groupes se rapprocher, l'un en donnant une femme, l'autre des biens - c'est la " compensation matrimoniale [bridewealth] ${ }^{8}$. Par l'intermédiaire des deux époux qui s'unissent sexuellement, ces groupes contribuent à la constitution du corps de nouveaux êtres humains ; le groupe des maternels pour le sang et la chair et celui des paternels pour les os. Dans ce système de pensée, les parents maternels sont les bénéficiaires d'un ensemble de prestations faites au cours de l'enfance, dont la justification explicite est souvent d'empêcher que les pouvoirs de vie dont ils sont les vecteurs ne se retournent en pouvoirs de mort s'ils éprouvaient une quelconque insatisfaction à l'égard de leurs neveux ou nièces. Au moment du décès de ceux-ci, ils reçoivent également des dons comme compensation pour les parties molles dont ils sont à l'origine et qui disparaissent à jamais.

Dans les substances, organes ou éléments osseux que le corps de chacun contient subsiste toujours un peu de celui - ici un groupe de parents - auquel il les doit. Ainsi, pour certains anthropologues, dont M. Strathern au premier chef $(1988$, p.131), les relations sont en fait les véritables éléments dont les personnes sont composées et l'accession à l'âge adulte, voire la vie entière, est une succession d'alternances entre des états relationnels spécifiques, transformés lors d'échanges ou de rituels au cours desquels « la composition relationnelle des personnes " est travaillée afin de satisfaire temporairement un objectif précis. M. Strathern écrit par exemple que, pour qu'un individu soit capable de se reproduire, il faut symboliquement éliminer en lui la partie corporellle issue de ses maternels pour un homme et de ses paternels pour une femme, afin d'atteindre un état corporel où les éléments sont tous entre eux dans une "relation de même sexe ». Ce raisonnement permettrait d'expliquer certains rites d'initiation masculine comprenant des pratiques d'expulsion de sang à la suite desquelles chaque garçon est symboliquement, mais efficacement, purgé du sang qu'il a hérité de sa mère (Strathern, 1988, p. 245).

Dans une perspective d'anthropologie comparative, on pourrait proposer que la vision occidentale, et dualiste, de la personne comme entité dotée d'un corps et d'une âme - ou pour parler de façon plus neutre, d'un corps et d'un moi (Théry et Bonnemère, 2008) favorise l'appréhension des nouveau-nés comme de véritables personnes. Dans une région comme la Nouvelle-Guinée, où ces deux attributs que sont le corps et l'esprit ne suffisent pas à faire d'un échantillon de l'espèce humaine une personne, et où c'est la capacité de celui-ci à répondre à la sollicitation d'autrui et à communiquer qui le font advenir tel, un nouveau-né tout juste sorti de la matrice, et même un bébé de deux semaines, ne répond pas à ce critère. II existe donc une période de latence pendant laquelle l'infanticide n'est pas conçu comme la mise à mort d'un congénère et devient donc moralement possible (Boltanski, 2004, p. 65). Cela n'implique pas qu'il soit pratiqué à tort et à travers car il ne devient pas pour autant un acte banal et facile à accomplir. Jamais sans doute il ne l'a été, nulle part et à aucune époque.

\section{Bibliographie}

${ }^{8} \mathrm{Au}$ risque de schématiser, on pourrait dire que la compensation matrimoniale est le symétrique de la dot. C'est donc ici la famille du mari qui « achète " une épouse et non celleci qui arrive chez son mari avec ses biens. 
Boltanski, L. 2004. La condition fœtale. Une sociologie de l'engendrement et de l'avortement, Paris, Gallimard.

Bonnemère, P. 1998. "Quand les hommes répliquent une gestation : une analyse des représentations et des rites de la croissance et de la maturation des garçons chez les Ankave-Anga (Papouasie-Nouvelle-Guinée)», in M. Godelier and M. Panoff (sous la direction de), La production du corps : approches anthropologiques et historiques, Amsterdam, Éditions des archives contemporaines, 81-113.

Bonnemère, P. 2008. "Du corps au lien: l'implication des mères dans les initiations masculines des Ankave-Anga ", in I. Théry and $P$. Bonnemère (sous la direction de), Ce que le genre fait aux personnes, Paris, Éditions de l'EHESS, 75-90.

Brown, G. 1910. Melanesians and Polynesians : Their life-histories described and compared, New York, Benjamin Blom, Inc. Publishers, 1972.

Butt, L. 1998. "The social and political life of infants among the Baliem valley Dani, Irian Jaya », PhD diss., Montreal, McGill University.

Godelier, M. 1982. La production des grands hommes, Paris, Fayard.

Lindenbaum, S. 1972. "Sorcerers, Ghosts and Polluting Women : An Analysis of Religious Belief and Population Control », Ethnology, 11(3), 241-253.

McDowell, N. (sous la direction de). 1988. Reproduction Decision Making and the Value of Children in Rural Papua New Guinea, Port Moresby, IASER.

Poole, J. P. P. 1985. "Coming Into Social being: Cultural Images of Infants in BiminKuskusmin Folk Psychology ", in G. M. White and J. Kirkpatrick (sous la direction de), Person, Self, and Experience: Exploring Pacific Ethnopsychologies, Berkeley, University of California Press, 183-242.

Read, K. 1955. "Morality and the concept of the person among the Gahuku-Gama », Oceania 25(4), 233-282.

Scheper-Hugues, N. 1992. Death Without Weeping: The violence of Everyday Life in Brazil, Berkeley, University of California Press.

Scrimshaw, S. C. M. 1984. "Infanticide in human populations: Societal and individual concerns ", in G. Hausfater and S. B. Hrdy, Infanticide: Comparative and Evolutionary Perspectives, New Brunswick and London, Aldine Transaction, 439-462.

Stasch, R. 2009. Society of Others: Kinship and Mourning in a West Papuan Place, Berkeley, University of California Press.

Strathern, M. 1988. The Gender of the Gift: Problems with Women and Problems with Society, Berkeley, University of California Press.

Théry, I. ; Bonnemère, P. (sous la direction de). 2008. Ce que le genre fait aux personnes, Paris, Éditions de l'EHESS (enquête 7).

Tillier, A. 2001. Des criminelles au village. Femmes infanticides en Bretagne (1825-1865), Rennes, P.U. de Rennes.

Valette-Cagnac, E. 2003. "Être enfant à Rome. Le dur apprentissage de la vie civique ", Terrain, 40, 49-64. 